\title{
Bath cooling analysis based on finite element method
}

\author{
Xinyu Jiang \\ North China Electric Power University, Beijing 102206, China \\ los963258741@163.com
}

Keywords: Finite element method, ANSYS, Three-dimensional temperature field

\begin{abstract}
To analyze the temperature field distribution of three-dimensional bathtub, the finite element method is applied. This is one of the very important parts of dealing the problem and establishing an appropriate model. Galerkin method of weighted residuals is used to obtain the variational equation of the three-dimensional temperature field. The bath water is discredited by using tetrahedral element and according to the third-class boundary conditions as well as the face very coordinates points transform law together with the initial conditions, all the temperature field are carefully simulated on ANSYS software. For transient temperature field, we use finite difference method to discredited the time-step and successfully solve the transient temperature field governing equation by replacing differentiation with difference method. The problem is surely a complicated one, so it is better to start with factors like air and water temperature, the shape and material of bathtub and location of faucet and overflow drain. Make reasonable assumption of some needed parameters at the same time. The model we build follows a process from easy to complicated. A model without human is built to examine the cooling and adding hot water process. By using finite element method, temperature distribution of the bathtub is cleared of the charge of time. Heat exchange and the influence of energy, momentum and flow between cold and hot water are also taken into account.
\end{abstract}

\section{Introduction}

Because of having little special circumstances and taking too much factors into account, the analysis of the temperature of the bathtub and heat transfer is complicated. This problem has factors like shape and material of the bathtub, the flow, temperature and spatial position of the water, the ambient temperature and the shape, temperature and movement of the person. Our goal is to start with an appropriate basic model and set it as the basis. Then with upgrade and refinement, we make our model satisfy those conditions and subtract factors that has too little influence on the result at the same time.

\section{Assumptions and Justifications}

The fluid matches the Bossiness assumptions ${ }^{[1]}$. The fluid viscous dissipation can be ignored. All the physical properties except density are constant.

For density, we only consider its change in the body force entries in momentum equation, others can be considered as constant.

\section{Formulation for finite elements equations ${ }^{[2]}$}

The formulation for finite elements equations has two ways: one is using variational approach. However, in most circumstances the differential equations cannot satisfy variational approach's condition, so it is impossible to formulate the pan function corresponding to the differential 
equation. The other one is the Galerkin method of weighted residuals, by which it is no need to find the pan function and the process of analyzing will be much easier. As a result, it is better to use the Galerkin method of weighted residuals.

For the following heat conduction equation which has a constant thermal conductivity

$\lambda\left(\frac{\partial^{2} T}{\partial x^{2}}+\frac{\partial^{2} T}{\partial y^{2}}+\frac{\partial^{2} T}{\partial z^{2}}\right)+q_{v}(x, y, z, t)-\rho C_{p} \frac{\partial T}{\partial t}=0$

Use Galerkin method to produce

$$
\mathrm{J}[T(x, y, z, t)]=\iiint_{\Omega} W_{\xi}\left[\lambda\left(\frac{\partial^{2} \tilde{T}}{\partial x^{2}}+\frac{\partial^{2} \tilde{T}}{\partial y^{2}}+\frac{\partial^{2} \tilde{T}}{\partial z^{2}}\right)-q_{v}+\rho C_{p} \frac{\partial \tilde{T}}{\partial t}\right] d x d y d z=0(\xi=1,2,3 \ldots n)
$$

where $\tilde{T}$ is the temperature field of the interpolation functions of some element, $\Omega$ is the three-dimensional solid solution domain of the temperature field and $W_{\xi}$ is Galerkin coefficient.

According to Galerkin method, define

$W_{\xi}=\frac{\partial \tilde{T}}{\partial T_{\xi}}(\xi=1,2,3 \ldots n)$

To avoid confusion, $\mathrm{T}$ will replace $\widetilde{T}$ below.

Use Green's theorem to relate the volume integration within area $\Omega$ to curved surface integration on the border $\partial \Omega$, and the following equation can be obtained

$\frac{\partial I}{\partial T_{\xi}}=\iiint_{\Omega} \lambda\left(\frac{\partial W_{\xi}}{\partial x} \frac{\partial T}{\partial x}+\frac{\partial W_{\xi}}{\partial y} \frac{\partial T}{\partial y}+\frac{\partial W_{\xi}}{\partial z} \frac{\partial T}{\partial z}\right) d x d y d z-\iiint_{\Omega} q_{v} W_{\xi} d x d y d z+\iiint_{\Omega} \rho C_{p} W_{\xi} \frac{\partial T}{\partial t} d x d y d z-$

$\oiint_{\partial \Omega} \lambda W_{\xi} \frac{\partial T}{\partial n} d s=0$

Where $I$ is a functional of temperature function $T$ and $\frac{\partial J}{\partial T_{\xi}}$ is a variational of the functional.

The equation above is the governing equation of three-dimensional temperature field calculation.

\section{Discrete structure}

Discrete structure is a serial of non-overlapping but continuous subdivided small areas divided from the researching area. These small areas are the finite elements. To get the solution to the finite element is to transfer the continuous solutions of the whole area into discrete solutions on the node.

For three-dimensional problems, it's a good way to regard them as tetrahedron and hexahedron. This essay uses regular grid-point. Use i, j, m, l to mark the internal points of each element. For the elements on the boundary, to simplify the calculation, the program in this essay allows there is a triangle face on the boundary and define that the boundary triangles are composed by node $\mathrm{j}, \mathrm{m}$, and $\mathrm{l}$ in order to oppose point $\mathrm{i}$.

\section{Determine the element interpolation function}

For the discretization of three-dimensional temperature field, when the element volume is 
appropriately small, the distribution of the temperature within the element can be approximately regarded as linear distribution. In this case a linear function can be selected as a temptation function. It is reasonable to consider temperature $T$ within the tetrahedral element is a linear function of the change of spatial coordinate $(x, y, z)$ :

$\mathrm{T}=a_{1}+a_{2} x+a_{3} y+a_{4} z$

Where $a_{1}, a_{2}, a_{3}, a_{4}$ are all undetermined coefficients. Substitute node point i, j, $\mathrm{m}$ and l of element "e" for the above equation and obtain:

$$
\left\{\begin{array}{c}
T_{i}=a_{1}+a_{2} x_{i}+a_{3} y_{i}+a_{4} z_{i} \\
T_{j}=a_{1}+a_{2} x_{j}+a_{3} y_{j}+a_{4} z_{j} \\
T_{m}=a_{1}+a_{2} x_{m}+a_{3} y_{m}+a_{4} z_{m} \\
T_{l}=a_{1}+a_{2} x_{l}+a_{3} y_{l}+a_{4} z_{l}
\end{array}\right.
$$

the interpolation function can be obtained:

$$
\begin{aligned}
& \mathrm{T}=\frac{1}{6 V_{i}}\left[\left(a_{i}+b_{i} x+c_{i} y+d_{i} z\right) T_{i}+\left(a_{j}+b_{j} x+c_{j} y+d_{j} z\right) T_{j}+\left(a_{m}+b_{m} x+c_{m} y+d_{m} z\right) T_{m}+\right. \\
& \left.\left(a_{l}+b_{l} x+c_{l} y+d_{l} z\right) T_{l}\right]
\end{aligned}
$$

Simplify the above equation as:

$$
\mathrm{T}=[N]^{e}\{T\}^{e}(8)
$$

In this equation $[N]^{e}=\left[N_{i}, N_{j}, N_{m}, N_{l}\right],\{T\}^{e}=\left\{N_{i}, N_{j}, N_{m}, N_{l}\right\}$

$$
N_{\xi}=\frac{1}{6 V_{\theta}}\left(a_{\xi}+b_{\xi} x+c_{\xi} y+d_{\xi} z\right)(\xi=i, j, m, l)
$$

The expression of $N_{\xi}$ only relates to the shape of the element, and it is a discretized shape function of three-dimensional element. (6) is the explicit form of this shape function. Express it as explicit function with coordinates $\mathrm{x}, \mathrm{y}$ and $\mathrm{z}$ :

$$
\left\{\begin{array}{l}
x=N_{i} x_{i}+N_{j} x_{j}+N_{m} x_{m}+N_{l} x_{l} \\
y=N_{i} y_{i}+N_{j} y_{j}+N_{m} y_{m}+N_{l} y_{l} \\
z=N_{i} z_{i}+N_{j} z_{j}+N_{m} z_{m}+N_{l} z_{l}
\end{array}\right.
$$

\section{calculate variation of the element}

Formulas of internal element, first class boundary element and thermal insulation element. For internal element, first class boundary element and thermal insulation element, the linear integration term are 0 . The variational equations have the same form:

$\frac{\partial I^{\varepsilon}}{\partial T_{\xi}}=\iiint_{\varepsilon} \lambda\left(\frac{\partial W_{\xi}}{\partial x} \frac{\partial T}{\partial x}+\frac{\partial W_{\xi}}{\partial y} \frac{\partial T}{\partial y}+\frac{\partial W_{\xi}}{\partial z} \frac{\partial T}{\partial z}\right) d x d y d z-\iiint_{\varepsilon} q_{v} W_{\xi} d x d y d z+\iiint_{\varepsilon} \rho C_{p} W_{\xi} \frac{\partial T}{\partial t} d x d y d z=0$

The result of the above formula can be presented by the following matrix after integration:

$$
\left\{\frac{\partial J^{\varepsilon}}{\partial T_{i}} \frac{\partial J^{e}}{\partial T_{j}} \frac{\partial J^{e}}{\partial T_{m}} \frac{\partial J^{\varepsilon}}{\partial T_{I}}\right\}^{T}=[K]^{e}\{T\}^{e}+[N]^{e}\left\{\frac{\partial T}{\partial t}\right\}^{e}-\{p\}^{e}
$$


Where $[K]^{e}$ is three-dimensional conduction matrix or conduction element stiffness matrix, $[N]^{e}$ is heat capacity matrix and $\{p\}^{e}$ is the rightmost vector.

The calculation of third class boundary element. Corresponding with tetrahedral element, the third class boundary element is the element which has one surface under the third class boundary conditions, that means, the heat transfer coefficient $a$ between one of the triangle surface and the adjacent fluid is known. Specify the third class boundary conditions by applying them into tetrahedral element's triangle jml and obtain the following formation:

$-\left.\lambda \frac{\partial T}{\partial \mathrm{n}}\right|_{\Delta \mathrm{jml}}=\alpha\left(T-T_{f}\right)$

\section{Time discretization of transient temperature field}

It is not enough to solve transient heat conduction equations only with a known boundary condition. In addition, the initial condition should be known, and time-step shall also be discretized. There are many usual difference methods, such as two-point forward method, two-point backward method, Crank-Nicolson method, Galerkin method, three-point backward method and so on. Because of not having steady problems, this essay uses backward difference method.

The basis equation of transient temperature field by using different difference methods is:

$$
\left(\sigma[K]+\frac{[N]}{\Delta t}\right)\{T\}_{t}=\left\{\frac{[N]}{\Delta t}-(1-\sigma)[K]\right\}\{T\}_{t-\Delta t}+\sigma\{P\}_{t}+(1-\sigma)\{P\}_{t-\Delta t}
$$

When $\sigma=1$, it is backward difference method;

When $\sigma=1 / 2$ it is Crank-Nicolson difference method;

When $\sigma=2 / 3$, it is Galerkin difference method;

When $\sigma=0$, it is forward difference method.

Starting from a specific temperature field initial value, choose a certain number of time-step and the distribution of the temperature at any time can be calculated by using these formulas.

\section{Results and analysis}

After the simulation by ANSYS ${ }^{[3]}$ we obtain curve of temperature of bath water and tub of the change of time. It is shown in the following figure. With analysis, the enclosed bathroom is a place with high humidity and low heat lost environment, so the change is not so noticeable. In ANSYS, the tub temperature is the outer face's temperature. The material of the tub is acrylic which has a good conservation of heat. 


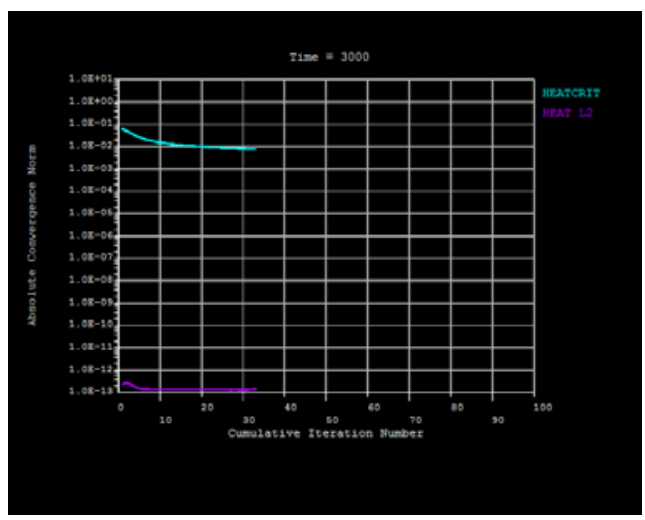

Figure 1 Temperature versus time

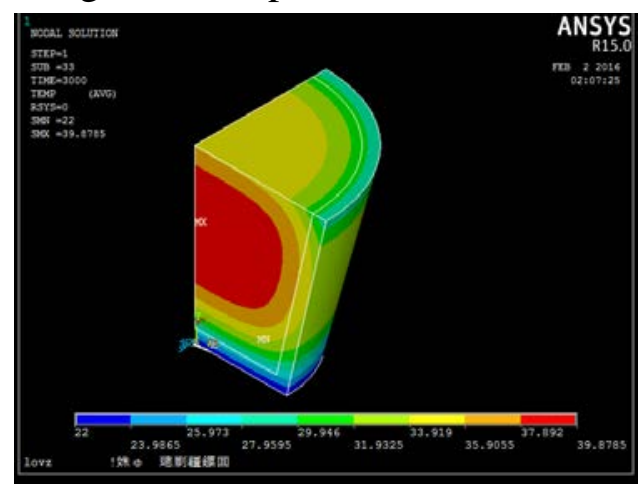

Figure 2 time in 3000s

In addition, we also obtain spatial temperature distribution of the tub at different time. Profile figures of the tub is below. Time is 528s, 860s and 3000s, respectively:

\section{Conclusions}

The thermal finite element model based on ANSYS platform, using heat transfer theory, through modeling and analysis, we found the hot bath cooling is not only related to the initial water temperature, but evaporation, bath materials and relative humidity will affect the temperature changes in the space on the field. This can be extended to the power plant or other research areas of water-cooling, so it has a broader significance in the practice

\section{References}

[1]Gray, D.D., and Giorgin, A., The Validity of the Boussinesqe Approximation for Liquids and Gases[M], Int.J.Heat Mass Transfer, 1976, 19: 545-551

[2] GU CHaohao\&LI Daqian \& CHEN SHuxing \& ZHEN Songmu \& TAN Yongji.Equations of Mathematical Physics[M].Beijing: Higher Education Press,2012.7

[3] Finite Element Analysis and Optimal Design Based on ANSYS in a XH2408 GantryStyle NC Machining Center[J]. International Journal of Plant Engineering andManagement,2010,03:188-192. 